\title{
Palliative surgery for cervical spine metastasis
}

\author{
Jai $\underline{R a O}^{1}$, MRCS, FRCSEd, Rajendra Tiruchelvarayan ${ }^{2}$, FRCS, FAMS, Lester $\underline{\text { Lee }}^{1}$, MBBS, MRCS
}

INTRODUCTION The present study aimed to assess the immediate/early clinical outcomes and surgical results of 11 consecutive patients who underwent palliative cervical spine surgery for symptomatic spinal metastases.

METHODS This single-surgeon retrospective case series analysed 12 surgical procedures that were performed for symptomatic cervical spinal metastasis in 11 consecutive patients. All surgeries were carried out at Singapore General Hospital, Singapore, from 2007 to 2013. Preoperative medical oncological assessment/staging was performed on each patient - all patients presented with either axial neck pain or neurological deficits, and had no bladder or bowel symptoms. The primary outcomes analysed were postoperative neurological power and improvement in neck pain.

RESULTS Anterior $(n=5)$, posterior $(n=4)$ and combined $(n=2)$ surgical approaches were used for decompression and stabilisation. Comparing between pre- and postoperative pain scores (scored according to the visual analog scale), and pre- and postoperative limb power scores (scored according to the Medical Research Council scale for muscle strength), we found that all patients showed improvement in their symptoms. Postoperatively, patients had either improvement or preservation of neurological power, and all patients had a decrease in axial neck pain after surgery. Although there was one case of minor pedicle screw instrumentation malplacement, this did not result in any neurological symptoms. Median survival for the patients was 108 (range 7-1,095) days.

CONCLUSION Palliative surgery for cervical spine metastasis is safe with good neurological results, low complication rates, and improvement in neck pain. A multidisciplinary approach involving surgeons, medical oncologists and radiotherapists is needed to optimise patient care and outcome.

Keywords: cervical spine, metastasis, palliative surgery

\section{INTRODUCTION}

The spinal column is the most common site for osseous involvement in metastatic disease, and spinal column involvement occurs in up to $70 \%$ of terminal cancer cases. ${ }^{(1-4)}$ Although cervical spine involvement (occurring in only $8 \%-25 \%$ of terminal cancer cases) is less common than thoracolumbar spine area involvement, ${ }^{(5)}$ metastatic disease in the cervical spine can result in increased morbidity due to the likelihood of spinal instability. It can also result in pain and neurological paralysis. Patients with pathological fractures of the cervical spine experience severe pain and require a period of bed rest. This may further increase the risk of medical complications such as pneumonia, bed sores and urinary tract infections. With the increasing incidence of cancer worldwide and better life expectancies secondary to improved treatment regimes, the incidence of bone metastases is expected to rise. ${ }^{(1,5)}$

The main goals of surgical intervention in spinal metastases are to: (a) relieve spinal cord compression; (b) provide spinal stability; (c) relieve symptomatic pain; and (d) obtain histological specimens for diagnosis. ${ }^{(1-3,6-8)}$ In previous studies conducted on cervical spine surgery for metastasis, questionnaires administered after surgery showed that patient satisfaction is directly related to improvement in neurological status, while family satisfaction is related to improvement in pain. ${ }^{(5)}$

The challenge with managing terminal cancer is balancing clinical improvement against the risks of surgery. There must also be a reasonable length of survival for the patient, based on the systemic metastasis disease burden, before surgery can be considered. In the present series we evaluated the outcomes of metastatic cervical spine patients treated with palliative surgery to assess the pain relief achieved with surgery, as well as the result of the surgeries. The surgical approaches used are herein discussed with the aid of a case report.

\section{METHODS}

This study was a retrospective single-surgeon surgical series conducted from 2007 to 2013 at the Department of Neurosurgery, Singapore General Hospital (SGH), Singapore. All patients underwent preoperative specialist oncological assessment. This preoperative oncological and surgical assessment involved a multidisciplinary team, which consisted of a medical oncologist, a radiation oncologist and a spinal neurosurgeon. Computed tomography (CT) of the chest, abdomen and pelvis was used to estimate each patient's prognosis. Surgical intervention was performed only if the estimated prognosis was greater than three months. Fitness for surgery was assessed using routine investigations such as blood tests (e.g. haemoglobin levels and clotting profile), plain chest radiography and electrocardiography. Specialist respiratory medicine, anaesthesia consults and preoperative lung function tests were obtained for patients with significant lung disease.

The primary aim of the surgery was to relieve spinal cord compression via surgical tumour debulking. This would lead to relief of intractable pain and provide good palliation with maintenance of the patient's ability to perform functional activities of daily living. The approach used was individualised based on 
Table I. Demographic and clinical characteristics of the patients $(n=11)$.

\begin{tabular}{|c|c|c|c|c|c|c|c|}
\hline Case & $\begin{array}{c}\text { Age at } \\
\text { operation (yrs) }\end{array}$ & Gender & $\begin{array}{l}\text { Primary tumour } \\
\text { location }\end{array}$ & $\begin{array}{l}\text { Primary preoperative } \\
\text { symptom }\end{array}$ & $\begin{array}{c}\text { Cervical } \\
\text { tumour level }\end{array}$ & $\begin{array}{c}\text { Visceral } \\
\text { metastasis }\end{array}$ & Surgical approach \\
\hline 1 & 66 & $\mathrm{~F}$ & Colon & Neck pain & $\mathrm{C7}$ & Yes & Anterior \\
\hline 3 & 69 & M & Prostate & Neck pain & $\mathrm{C} 6$ & No & Posterior \\
\hline 4 & 22 & M & Nasopharynx & Limb weakness & $\mathrm{C} 6 / \mathrm{C} 7$ & No & Posterior \\
\hline 5 & 35 & M & Liver & Neck pain & $\mathrm{C} 3$ & Yes & Anterior + posterior \\
\hline 6 & 54 & M & Lung & Neck pain & $\mathrm{C} 2+\mathrm{C} 7$ & Yes & Anterior \\
\hline 7 & 65 & M & Liver & Neck pain & C6 & No & Posterior \\
\hline 8 & 49 & M & Lung & Neck pain & $\mathrm{C} 3+\mathrm{C} 6$ & Yes & Anterior + posterior \\
\hline 9 & 48 & $\mathrm{~F}$ & Colon & Neck pain & $\mathrm{C7}$ & Yes & Anterior \\
\hline 10 & 55 & M & Liver & Neck pain & C3 & No & Anterior \\
\hline 11 & 49 & $\mathrm{~F}$ & Kidney & Neck pain & $\mathrm{C} 4 / \mathrm{C5}$ & Yes & Posterior \\
\hline
\end{tabular}

F: female; M: male

whether the spinal cord/nerve roots could be decompressed, as well as whether there was a need for spinal stabilisation.

Patients with tumours that either predominantly involved the vertebral body or caused a pathological fracture underwent an anterior approach, which consisted of cervical corpectomy and tumour debulking with anterior cervical plating. The choices for corpectomy replacement were either an iliac crest bone graft (done in one patient) or a titanium mesh cage strut. If the tumour was predominantly dorsal to the spinal cord, a posterior approach was used, which consisted of cervical laminectomy and tumour debulking with titanium posterior instrumentation fusion. Either lateral mass or pedicle screws were used for instrumented fusion. Spinal neuronavigation using intraoperative CT was performed in cases where there was abnormal/complicated cervicothoracic bone anatomy requiring additional screw guidance. Postoperative adjuvant radiotherapy was planned for all patients and adjuvant chemotherapy was used for chemotherapy-sensitive tumours, as decided by the medical oncologists.

The primary outcome assessed was immediate postoperative neurological power and pain relief. Neurological power was assessed by comparing the immediate postoperative limb power scores at postoperative Day 1 against the preoperative scores. The Medical Research Council (MRC) scale for muscle strength (ranging from 0 to 5) was used.(9) Pain relief was assessed based on the patient's description of the level of pain, using the visual analog scale (VAS), which has a score scale of $0-10$. Secondary outcomes assessed were: (a) development of surgical wound infection; (b) instrumentation failure/ malplacement; (c) estimated intraoperative blood loss; and (d) overall patient survival.

\section{RESULTS}

A total of 12 cervical decompression surgeries for spinal metastases were performed for 11 consecutive patients (8 men, 3 women) at SGH from 2007 to 2013. The demographic and clinical characteristics of the patients are shown in Table I. The average age at surgery was 52.2 (range 22-69) years. All patients were symptomatic at the time of surgery - 10 patients presented with complaints of axial neck pain, and 8 patients, with
Table II. Preoperative and immediate postoperative Medical Research Council scores for muscle strength ${ }^{(9)}$ of the patients $(n=11)$.

\begin{tabular}{|c|c|c|c|c|c|c|c|c|}
\hline \multirow[t]{3}{*}{ Case } & \multicolumn{4}{|c|}{ Preoperative } & \multicolumn{4}{|c|}{ Postoperative } \\
\hline & \multicolumn{2}{|c|}{ Upper limbs } & \multicolumn{2}{|c|}{ Lower limbs } & \multicolumn{2}{|c|}{ Upper limbs } & \multicolumn{2}{|c|}{ Lower limbs } \\
\hline & Right & Left & Right & Left & Right & Left & Right & Left \\
\hline 1 & 4 & 4 & 5 & 5 & 4 & 4 & 5 & 5 \\
\hline 2 & 2 & 5 & 5 & 5 & $3 *$ & 5 & 5 & 5 \\
\hline 3 & 4 & 5 & 5 & 5 & 4 & 5 & 5 & 5 \\
\hline 4 & 5 & 5 & 2 & 4 & 5 & 5 & $3^{*}$ & 4 \\
\hline 5 & 3 & 5 & 5 & 5 & 3 & 5 & 5 & 5 \\
\hline 6 & 4 & 4 & 4 & 4 & 4 & 4 & 4 & 4 \\
\hline 7 & 5 & 5 & 5 & 5 & 5 & 5 & 5 & 5 \\
\hline 8 & 5 & 5 & 5 & 5 & 5 & 5 & 5 & 5 \\
\hline 9 & 4 & 3 & 5 & 5 & 4 & 3 & 5 & 5 \\
\hline 10 & 4 & 5 & 5 & 5 & 4 & 5 & 5 & 5 \\
\hline 11 & 4 & 5 & 5 & 5 & 4 & 5 & 5 & 5 \\
\hline
\end{tabular}

*Improved from preoperative score.

complaints of neurological weakness. No patient complained of urinary or bowel dysfunction.

Four patients had multilevel disease at the time of presentation - two had adjacent level involvement at $\mathrm{C} 6 / \mathrm{C} 7$ and C4/C5, while two had remote vertebral involvement at C3/C6 and $\mathrm{C} 2 / \mathrm{C} 7$. Five patients underwent anterior corpectomy with plating, four had posterior laminectomy with instrumentation, and two underwent both anterior and posterior surgery. Of the two patients who underwent combined anterior and posterior surgery, one had a staged surgery five days prior. Thus, a total of 12 surgical procedures were performed in the 11 patients included in the present study.

Neurological deficit due to the surgical procedure performed was not observed in any of the 11 patients (Table II). At the time of discharge, while the neurological power of eight patients was unchanged, it was found to be improved in three patients. All patients experienced reduced axial neck pain, according to the patients' postoperative VAS scores (Table III) taken at follow-up one month after surgery (except for Case 6, as the patient died one week after the surgery due to a bowel perforation). One patient (Case 7) developed increased axial neck pain one year 

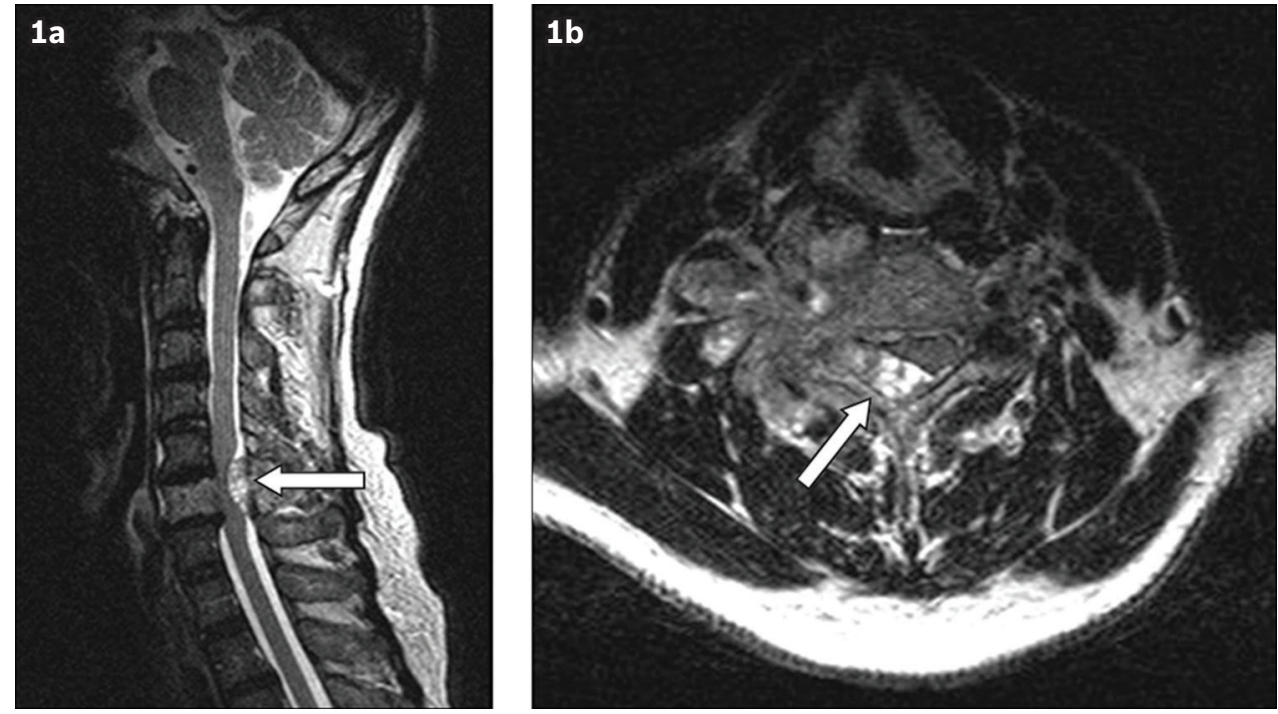

Fig. 1 T2-W (a) sagittal and (b) axial MR images of the cervical spine show severe epidural cord compression by a tumour at the $\mathrm{C} 6$ level (arrows). The tumour is predominantly dorsal and to the right of the spinal cord. The lamina and vertebral bodies are also infiltrated by the metastatic tumour.

Table III. Pain scores based on the visual analog scale (VAS), length of hospitalisation and duration of follow-up of the patients $(n=11)$.

\begin{tabular}{lccccc}
\hline Case & \multicolumn{2}{c}{ VAS pain score } & & \multicolumn{2}{c}{ Length (days) } \\
\cline { 2 - 3 } \cline { 5 - 6 } & Preoperative & Postoperative & & Hospitalisation & Follow-up \\
\hline 1 & 10 & 2 & & 11 & 86 \\
2 & 3 & 0 & & 45 & 96 \\
3 & 2 & 0 & & 15 & 330 \\
4 & 0 & 0 & & 17 & 596 \\
5 & 4 & 0 & & 11 & 111 \\
6 & 7 & 6 & & 22 & 7 \\
7 & 3 & 0 & & 11 & 739 \\
8 & 10 & 8 & & 16 & 87 \\
9 & 3 & 2 & & 79 & 63 \\
10 & 2 & 0 & & 4 & 20 \\
11 & 1 & 0 & & 23 & 38 \\
\hline
\end{tabular}

after surgery due to a new pathological fracture at a site remote to the level that was treated. The median length of hospitalisation was 16 (range 4-79) days and the median follow-up period was 87 (range 7-739) days. At the time of writing, the median survival period was 108 (range 7-1,095) days.

Spinal neuronavigation was used in six patients, when instrumentation involved the cervicothoracic junction. In our operating theatre facility, intraoperative CT scanners are used for navigation and postoperative surveillance of implant placement. The majority of the instrumentation was inserted with good results, with only one pedicle screw lateral breakout at the T1 level. The pedicle screw lateral breakout, which was detected on intraoperative $\mathrm{CT}$, resulted in no observable clinical consequence. One patient suffered malalignment of the anterior plates and screws five months after surgery due to progression of her original cervical metastasis. The malalignment was treated with a hard cervical collar. Thereafter, the patient did not develop new neurological deficits or pain.

Neither wound infection nor dehiscence was seen in any of the 11 patients. Data on estimated intraoperative blood loss was available for 9 of the 12 surgical procedures; intraoperative blood loss averaged 600 (range 200-1,250) mL per operation. One patient died one week after surgery secondary to acute bowel perforation, which was not related to the primary disease or the treatment of the disease. There were no other perioperative deaths. The surgical approach used is discussed in the following case.

A 69-year-old Chinese man, who was first diagnosed with prostatic cancer (Gleason score 4+5) in 2003, was on antiandrogen therapy. He had an unremarkable skeletal survey in 2007. He subsequently defaulted on his existing medical treatment to seek treatment using traditional medicine. In May 2008, he presented with a three-month history of neck pain and right-sided weakness that had persisted for one week. Physical examination revealed tenderness at the lower neck, which resulted in a reduced range of motion. Neurological examination showed mild upper right limb weakness with power grade 4, and decreased sensation in the right $\mathrm{C} 5$ and $\mathrm{C} 6$ dermatome; anal tone was intact. Magnetic resonance (MR) imaging of the cervical/thoracic spine (Fig. 1) was performed and severe epidural cord compression at the C6 level was observed. The tumour was predominantly dorsal and to the right of the spinal cord, and was compressing the right C6 nerve root at the exit foramen. The lamina and vertebral body were also infiltrated, but no pathological compression fracture was observed. The other levels of the cervical spine were unremarkable.

In view of this particular patient's medical history, the lesion was deemed to likely be metastatic. CT of the patient's thorax/ abdomen/pelvis showed no visceral metastasis. After medical oncological staging and preoperative surgical assessment for anaesthetic fitness, the patient underwent C5/C6 laminectomy with surgical debulking of the tumour and $\mathrm{C} 4-\mathrm{T} 2$ posterior spinal instrumentation. Lateral mass screws were inserted in C4 and C5, while pedicle screws were inserted into T1 and T2. These were connected by two dual-diameter rods and two cross-links were used for translational stability (Fig. 2). There were no postoperative complications, and the patient underwent postoperative physiotherapy. He was discharged well with no 

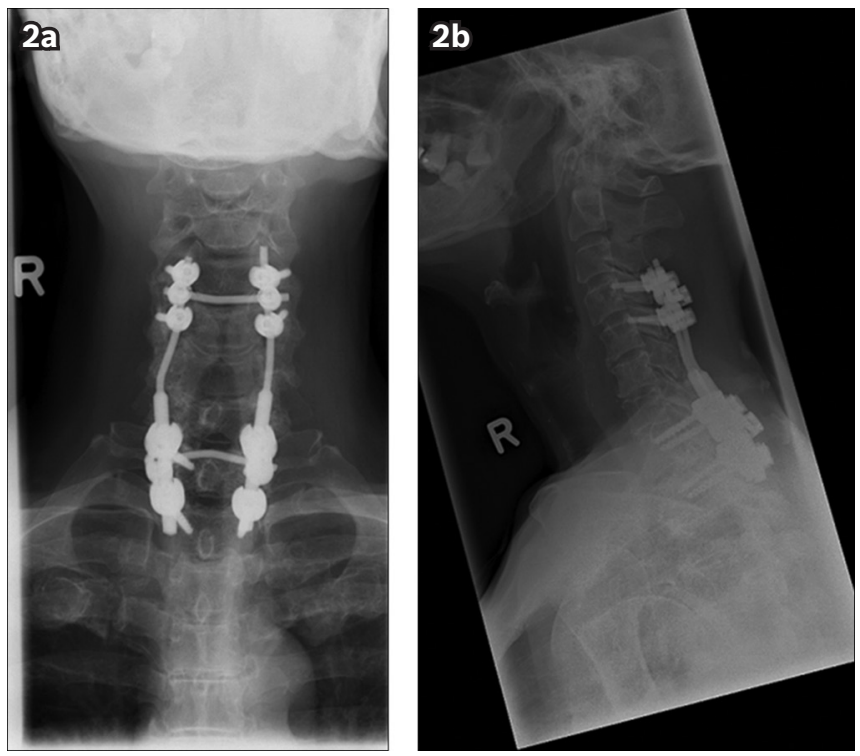

Fig. 2 (a) Anterior-posterior and (b) lateral plain radiographs of the cervical spine show the final cervicothoracic construct.

neck pain. Adjuvant radiotherapy (dose of 30 Gy) was given two weeks after discharge; wound inspection was done prior to the commencement of radiotherapy to ensure adequate wound healing. At the time of writing, this patient was stable and regularly attending outpatient medical follow-up.

\section{DISCUSSION}

As curative surgery is usually not possible in patients with symptomatic metastatic spinal disease, it is important to consider palliative surgery as an option. Although surgery is historically performed to prolong survival, there has been a paradigm shift in recent years to focus on the benefits of surgery in improving symptoms that negatively affect the quality of life of terminally ill patients. ${ }^{(2,10)}$ Patients with symptomatic metastasis commonly experience axial pain, radicular pain and neurological dysfunction. ${ }^{(1-3,6-8)}$ In our 11 patients, the majority of the patients presented with severe disabling neck pain that limited their ability to carry out activities of daily living.

The aim of surgical treatment in the present case series was to provide pain relief (i.e. reduction of axial neck pain) with minimal morbidity to improve the quality of life of the patients. Despite the complexities involved in spinal surgery, none of our patients suffered adverse neurological deterioration immediately after surgery. This shows that surgery can be carried out effectively at the cervical spine with minimal neurological morbidity.

The resolution of pain symptoms allows for the early mobilisation of patients, and thus by extension, the early institution of physiotherapy. When intensive physiotherapy is instituted early, there will be some chance for recovery and improvement in neurological power. At the time of discharge, 3 of our 11 patients had improved neurological power postoperatively. Without treatment, the continued neurological deterioration of patients may lead to an increased risk of pneumonia and bed sores, due in part to the immobility associated with severe cord compression. Surgery to maintain the neurological function of patients could also be construed as palliative, as it helps patients maintain a level of functional activity, which in turn decreases the risk of concomitant infections and the need for further hospitalisation.

While surgical decompression has not been proven to directly improve functional outcome, studies have shown improvement in functional scores, with improvement maintained for at least one year after surgical intervention. ${ }^{(2)}$ As pain inhibits activity, it follows that the reduction of pain would commensurate with an increase in activity. Furthermore, the maintenance of neurological function for a longer period of time can be achieved with surgical debulking of the tumour to reduce the likelihood of early cord compression.

Patients with no neurological compromise and no evidence of instability are usually referred for nonsurgical treatment, such as radiotherapy. ${ }^{(2,3,11)}$ In these cases where the primary presenting symptom is pain, there is usually a return of symptoms within six months after treatment. ${ }^{(11)}$ In the present case series, which evaluated patients who were surgically treated, only 1 of the 11 patients suffered a recurrence of axial pain. The recurrent axial pain in this patient was secondary to a new pathological fracture at a site remote to the original targeted level. This recurrence of pain after surgery occurred at a substantially later time (almost 12 months), compared to the time to pain recurrence in historical nonoperative treatments (within six months). Our findings suggest that surgery should be considered an option in the treatment algorithm for patients when patient survival is likely to exceed three months. If a patient who had previously undergone radiotherapy develops recurrent pain or neurological deficits after radiotherapy, surgery can also be considered.

With respect to medication for neck pain, it is noteworthy that the significant cervical pain may result in the need for high doses of opioids. Long-term usage may have side effects such as constipation and increased somnolence, negatively affecting patients' quality of life at an early stage. Findings from previous studies $^{(1)}$ and the present case series suggest that surgery can possibly provide the benefit of reduced analgesic usage via the relief of axial neck pain.

The surgical approach used depends on tumour topography - a predominantly anterior tumour is best approached anteriorly, and a posteriorly located tumour is best approached posteriorly. The surgical approach is chosen with the aim of achieving maximal tumour debulking. The goals of surgery are to: (a) provide pain relief; (b) reduce the likelihood of further cord compression; (c) restore spinal stability; and (d) obtain histological specimens for diagnosis. Since tumours that metastasise to the subaxial spine (C3-C6) tend to involve the anterior column due to marrow infiltration of the vertebral body, the predominant procedure of choice is an anterior approach in the subaxial spine. ${ }^{(3)}$ This usually involves corpectomy with reconstruction using a titanium cage or an iliac crest bone strut.

In the case report included, we highlighted a circumstance in which a posterior approach was required. The MR images of the patient showed that the cord compression was dorsal to the spinal cord. Also, despite the marrow involvement of the C6 vertebral body, there was no pathological fracture observed. These two 
factors - the location of the compression and the need/lack of need for stabilisation - are important in deciding whether the surgery should be done using the anterior or posterior approach. In our highlighted patient, we used a posterolateral approach, and significant tumour debulking was achieved together with good visualisation of the C6 nerve root, which was encased by the expanding mass. In this particular patient, maximal surgical benefit was achieved via a single approach rather than a combined approach. As the aim of palliative surgery is symptom relief, we believe that patients should undergo the surgical procedure that has the least risk, in order to achieve that aim.

Unfortunately, some patients would require both anterior and posterior approaches for surgical treatment to be effective. The combined (anterior and posterior) approach was used in 2 of our 11 patients. If the patient documented in our case report had a pathological fracture of the C6 vertebral body, he/she would also require the combined approach; the anterior approach would be needed to restore the height of the anterior column. On its own, a posterior construct would not be able to sufficiently prevent further kyphosis in the future. Further kyphosis would lead to complaints of neck pain, and thus, the failure of one of the primary aims of the surgical treatment.

There are risks associated with surgery, including damage to the spinal cord, haemorrhage and infection. In the present series, we minimised postoperative complications through the use of a standard protocol, which is summarised in the following text. We believe that the use of these techniques will help to ensure low rates of complications, as was demonstrated in the present case series.

1. To minimise the risk of a surgical site infection, all our patients undergo preoperative baths with $4 \%$ chlorhexidine gluconate. Chlorhexidine solutions have been shown to be superior to soap-based solutions in reducing bacterial colony counts in the axilla and groin of healthy volunteers. ${ }^{(12)}$ All of our patients are optimised preoperatively via chest physiotherapy and improved nutritional intake, with the assistance of our department's allied health staff. Intraoperative infection risks are minimised by reducing movement in and out of the operating theatre. Surgical gloves are also routinely changed prior to handling spinal implants.

2. Spinal navigation is routinely used when implants are needed at the cervicothoracic junction. Our operating theatre facility is equipped with an intraoperative, CT-based, computer guidance system. The use of this system is advantageous when compared to the use of image intensifier radiography. At certain anatomical regions such as the lower cervical spine/cervicothoracic junction, the patient's shoulders obstruct the lateral radiographic beam, leading to difficulty in visualising spinal screw placement. This problem is not present with CT imaging. With the aid of CT-based spinal navigation, we are able to plan the entry points and trajectories of the screws, as well as allow for real-time correction. The risk of injuring the spinal cord is reduced as we are able to avoid medial pedicle breakouts. (As described in the Results section, one of our patients did have a lateral breakout of a pedicle screw at the T1 level. This, however, was a minor breach with no clinical consequence. Screw revision was not necessary in that case.) Spinal navigation is also beneficial in patients with abnormal cervical anatomy secondary to tumour destruction of the normal anatomy. Intraoperative imaging of patients who are at this stage would allow surgeons to have a high degree of confidence when planning screw entry points and trajectories.

3. Postoperative systemic complications such as pneumonia and deep venous thrombosis are best reduced with early mobilisation. The early resolution of pain allows for intensive physiotherapy to be commenced earlier. Patients are encouraged to mobilise early and are also given incentive spirometry to reduce basal atelectasis, and thereby also reducing the risk of pneumonia. Early mobilisation also has the beneficial effect of reducing direct pressure on a posterior surgical wound. This allows for early wound healing and thus lowers the risk of wound infection.

In conclusion, palliative surgery of the cervical spine should be considered in appropriately selected patients. The present case series shows that palliative surgery of the cervical spine can achieve good results. It is a safe palliative treatment with low complication rates and good neurological results. Good palliation is achieved with a reduction in axial neck pain. A multidisciplinary team approach consisting of medical oncologists, spine surgeons and radiotherapists will help to ensure optimal patient outcome.

\section{REFERENCES}

1. Bartels RH, van der Linden YM, van der Graff WT. Spinal extradural metastasis: review of current treatment options. CA Cancer J Clin 2008; 58:245-59.

2. Quan GM, Vital JM, Pointillart V. Outcomes of palliative surgery in metastatic disease of the cervical and cervicothoracic spine. J Neurosurg Spine 2011; 14:612-8.

3. Oda I, Abumi K, Ito M, et al. Palliative spinal reconstruction using cervical pedicle screws for metastatic lesions of the spine: a retrospective analysis of 32 cases. Spine (Phila Pa 1976) 2006; 31:1439-44.

4. Bartels RH, Feuth T, van der Maazen R, et al. Development of a model with which to predict the life expectancy of patients with spinal epidural metastasis. Cancer 2007; 110:2042-9.

5. Sciubba DM, Gokaslan ZL. Are patients satisfied after surgery for metasttic spine disease. Spine J 2010; 10:63-5.

6. Fehlings MG, David KS, Vialle L, et al. Decision making in the surgical treatment of cervical spine metastases. Spine (Phila Pa 1976) 2009; 34:S108-17.

7. Marchesi DG, Boos N, Aebi M. Surgical treatment of tumors of the cervical spine and first two thoracic vertebrae. J Spinal Disord 1993; 6:489-96.

8. Jónsson B, Jónsson H Jr, Karlström G, Sjöström L. Surgery of cervical spine metastases: a retrospective study. Eur Spine J 1994; 3:76-83.

9. Medical Research Council. Aids to examination of the peripheral nervous system. Memorandum no. 45. London: Her Majesty's Stationery Office, 1981.

10. Kim A, Fall P, Wang D. Palliative care: optimizing quality of life. J Am Osteopath Assoc 2005; 105:S9-14

11. Rao S, Badani K, Schildhauer T, Borges M. Metastatic malignancy of the cervical spine. A nonoperative history. Spine (Phila Pa 1976) 1992; 17:S407-12.

12. Tanner J, Gould D, Jenkins $P$, et al. A fresh look at preoperative body washing. J Infect Prev 2012; 13:11-5. 